\title{
4 \\ Session Management and Control for Intelligent Multimedia Networks
}

\author{
O. Schreyer, St. Abramowski, T. Helbig, U. Konrads, K. Neunast
}

Philips Research Laboratories

P.O. Box 1980, D-52021 Aachen, Germany

Tel.: +49.241.6003.573, Fax: +49.241.6003.518

e-mail: schreyer@pfa.research.philips.com

\begin{abstract}
State-of-the-art in implementing multimedia applications is their development "from scratch". Application programmers have to re-implement common functions, such as communication control, anew for each multimedia application. To overcome the inefficiency, functions common to different networked multimedia applications (the so-called middleware) should be provided via application-oriented programming abstractions. Based on our Multimedia Reference Model as an overall conceptual framework we focus on an important middleware part, called session management and control (SMC). It realizes functions to unify and simplify the usage of data processing and data transfer capabilities of the basic communication and operating systems. We explain concepts and motivation of the basic SMC functionality and illustrate them by an example scenario.
\end{abstract}

\section{Keywords}

Multimedia, middleware, session management and control, application programming interface, stream control, quality of service 


\section{INTRODUCTION}

Advances in computer and communication technology have enabled the integration of digital audio and video in various fields of applications, leading to the emergence of so-called multimedia applications. Current multimedia applications are either local, single-user systems like computer games and CD-i applications or, if networked, restricted to a certain class of applications like video-on-demand, or tele-shopping. In the future, networked multi-user multimedia applications with a high degree of interactivity will emerge. Examples for such applications are networked tele-diagnosis, tele-teaching or tele-collaboration applications, as well as distributed multi-user games, or news editing tools for digital TV studios with distributed components like journalist workstations, video servers, and special effect rendering machines.

State-of-the-art in implementing multimedia applications is their development "from scratch", i.e. directly on top of basic communication and operating system functions. Application programmers have to re-implement common functions, such as communication control or quality of service handling, anew for each multimedia application instead of being able to concentrate on the core application logic itself. This is time consuming and inefficient. The solution is obvious: Functions common to different networked multimedia applications should be provided via applicationoriented programming abstractions.

These application-oriented functions (often referred to as middleware) close the gap between the functionality offered by basic communication and operating systems and the requirements of interactive, distributed multi-user multimedia applications. The following generic functions are among the essential ones needed for networked multi-user multimedia applications:

- configuring of multimedia communication and processing topologies,

- communication and coordination of multiple concurrent application entities,

- negotiating quality of service requirements and reserving resources,

- controlling and synchronizing data streams.

For complex systems, such as networked multimedia systems, an overall conceptual framework is strongly needed that allows to offer different views to end users, application designers, providers, and operators. In chapter 2 we shortly discuss our Multimedia Reference Model.

Session management and control is an important middleware part in this model covering the usage of data processing and data transfer capabilities of basic communication and operating systems. In chapter 3 we explain the basic terminology to embed SMC into the context of multimedia systems.

For the cooperation with applications we developed a session management and control application programming interface focused on in this paper. In the main part we explain motivation and concepts of the different groups of SMC operations. Finally we show the usage by an example scenario. 


\section{THE MULTIMEDIA REFERENCE MODEL: A FRAMEWORK}

For complex systems, such as networked multimedia systems, an overall conceptual framework is strongly needed. Different views have to be offered to end users, application designers, providers, and operators. In [3] we introduced the Multimedia Reference Model (see figure 1) by applying the modelling technique of the Intelligent Network Conceptual Model (INCM) based on our experiences with the realization of IN concepts (as shown e.g. for the service creation environment PHIDES [1] and the Open Switching platform [2]).

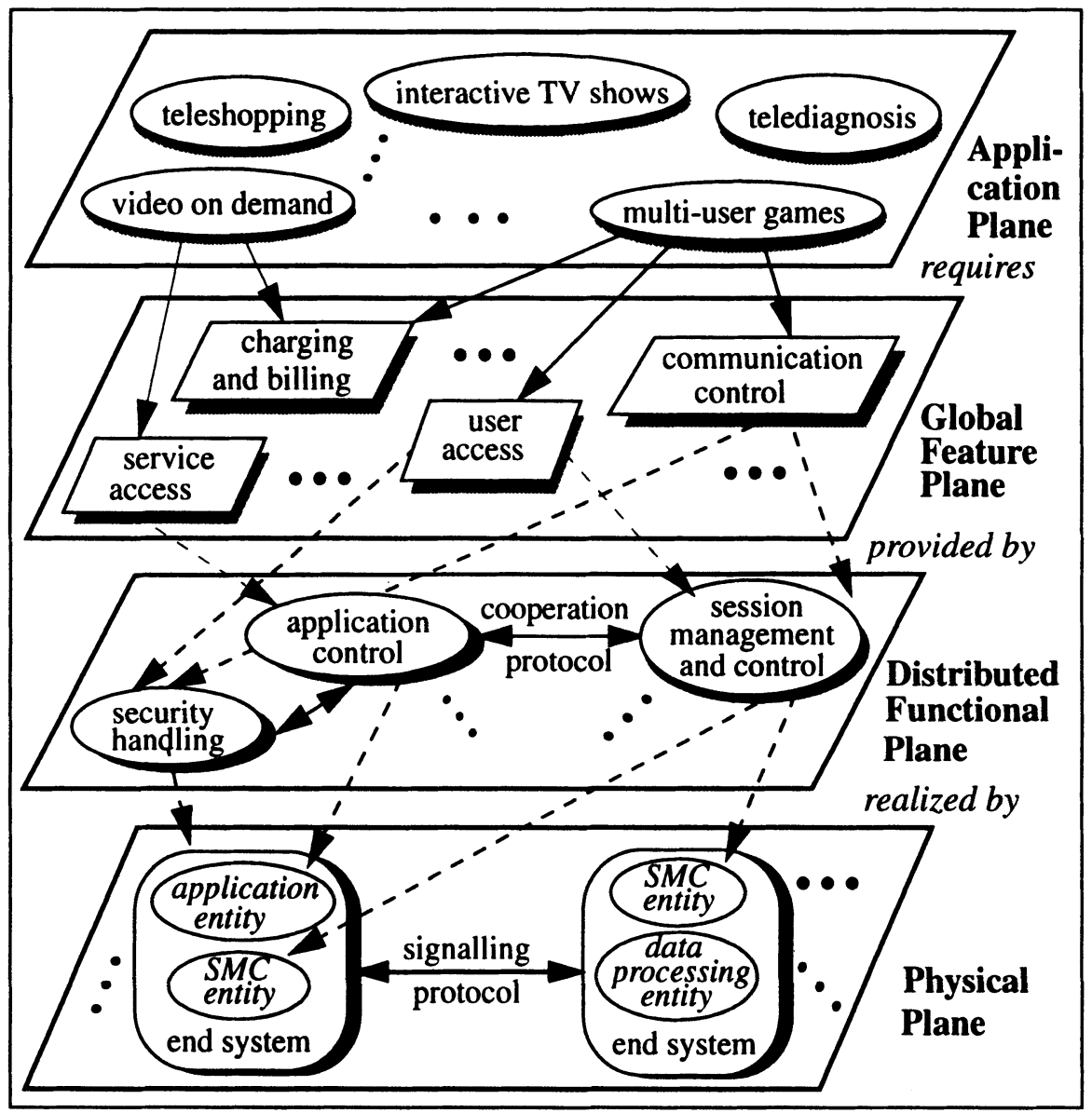

Figure 1: Multimedia Reference Model

The Application Plane describes the end user's view to the offered applications. Examples for such applications are tele-diagnosis, tele-shopping, interactive TV shows, video on demand, or multi-user games. 
The Global Feature Plane defines the application building blocks (ABB) necessary to construct the networked, interactive, multi-user, multimedia applications described on the Application Plane. Those ABBs are combined to construct the application logic. The basic $\mathrm{ABB}$ for underlying multimedia communication is the communication control ABB.

The Distributed Functional Plane (DFP) describes a functional architecture constructed of functional entities and their cooperation protocols, which are needed to provide the functionality for the application building blocks of the global feature plane. Session management and control is a basic functional entity.

The Physical Plane describes possible distribution scenarios that can reach from centralized realization of single-vendor solutions to the maximum distribution where every functional entity of the DFP is realized on one physical end-system.

\section{BASIC TERMS OF SESSION MANAGEMENT AND CONTROL}

As mentioned above, application-oriented (middleware) functions offered via a generic application programming interface enable application programmers to efficiently use the basic capabilities of the physical network in their applications without detailed knowledge of the implementation or of the intrinsics of the underlying communication infrastructure.

Session management and control (SMC) is an important middleware function on the DFP in our model. It covers the functionality to unify and simplify the usage of data processing and data transfer capabilities of the physical network components ("physical layer"). That means, the SMC is a distributed software on top of the basic communication and operating systems forming the physical layer. The management part of SMC refers to functions to configure data processing and data transfer entities in the sense of making them known to SMC, defining their attributes, or removing them from the control of SMC. The control part of SMC is concerned with functions provided to unify and simplify the runtime control of these entities.

The term application denotes software in the overall system that invokes SMC (middleware) functions during run-time to trigger and control the required data processing and data transfer actions in the physical network components. In the DFP, applications are represented by application control functions (ACFs) that are application parts realizing the interaction with the middleware. An ACF is considered to be an addresseable piece of software allocated on a single, well-defined end-system (see below). Each ACF may act on behalf of a user. However, in view of SMC, a user is (only) represented by a unique identity in relation to the ACF

The total amount of SMC functions provided to ACFs is collected in the session management and control application programming interface (SMC-API). The interaction between SMC and ACFs, consisting of SMC function invocations from the applications and their corresponding responses as well as possible notifications 
from the SMC, is defined by the session management and control application protocol (SMCAP).

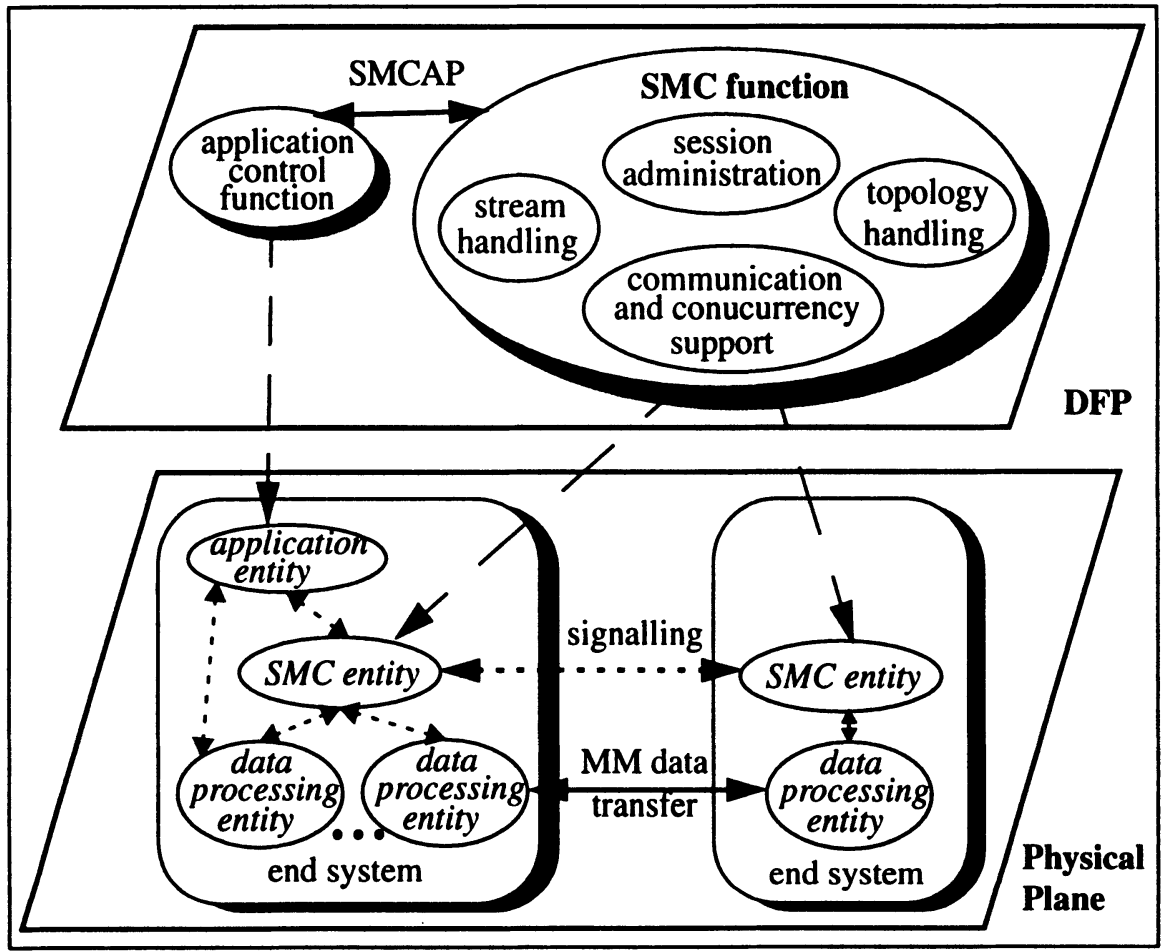

Figure 2: Placement of the SMC-API in Multimedia Reference Model

To keep the relationship between ACF/SMC interactions, the session concept as administrative wrapper for an (application) context is introduced. For this, the SMC-API contains functions to create, protect, modify, and release - i.e. to administrate - sessions (see chapter 4.1). For each session, a set of data called session profile is stored and maintained by SMC.

In figure 2 the embedding of the SMCAP in the DFP is shown. In the physical plane, we consider networks consisting of interconnected end-systems. End-systems are well-defined, separated, and addresseable pieces of hardware where both the software realizing the data processing and data transfer functions and the SMC software is running and/or the software realizing the ACFs. Examples for endsystems can be a PC connected to the network as well as a set-top box. An application entity should normally communicate with a local (or at least a welldefined) SMC entity. 


\section{SMC-API OPERATIONS}

In the following, we explain motivation and concepts of the different groups of SMC-API operations. These are:

- functions to administrate (i.e. create, inquire, etc.) sessions (chapter 4.1),

- functions to handle multimedia processing topologies (chapter 4.2),

- functions to negotiate quality of service contracts (chapter 4.3),

- stream handling functions to control data transfer and processing during runtime (chapter 4.4),

- further, only shortly considered function groups are (chapter 4.5)

- functions for application concurrency and coordination support,

- functions to support communication among application entities,

- functions to access the operational interfaces of processing entities,

- functions for session event handling,

- SMC management.

An example scenario (chapter 5) concludes this overview.

\subsection{Session Administration Operations}

A basic SMC task is storing and maintaining the context in which its functions are called. For this, the session concept as administrative wrapper for the relationship between all interactions concerning this context is introduced. Based on this concept, the following SMC functionality has to be provided:

- Creating a session

A session is created after a corresponding request from an ACF. In response, the SMC sends back the unique session identifier to be the common identification means for all interactions between ACFs and SMC belonging to the same (session) context. The ACF may optionally provide a session name by which the session can be identified by other ACFs without knowing the session identifier. The ACF creating a session becomes its session master. The role of the session master (and the related rights) may be passed and shared among ACFs. There must always be at least one session master. A session without any session master (e.g. if the (last) session master leaves the session by error) is automatically shut down by SMC.

- Adding, removing, and authenticating participants of sessions

An ACF is a participant of a session if it has been explicitly registered. Multiple ACFs can participate in a session. As soon as the session is created by the session master ACF (this is the first participant) and as long as the session exists, other ACFs may enter the session. The actual list of participants of a session is maintained in the session profile. An end-system is considered to be participating in a session as soon as one ACF located on the end-system is participating in the session. Removing an end-system from a session means to remove all ACFs located on this end-system from the session. 


\section{- Inquiring sessions}

In general, an ACF can query SMC concerning information about sessions and their attributes with or without being registered in any session. Inquiries may concern all sessions in progress (session directory), sessions on a specific end-system or sessions where a specific ACF is participating in etc. The SMC-API may offer inquiry functions. Another solution could be read-only interface to the session profile database.

- Protecting sessions

The execution of an SMC function (requested by an ACF) may be protected depending on the status of the calling ACF/user and the status of the session. Protection procedures (as password authentication or check of global rights or credit limits) are performed by a security handling function that is an function outside $S M C$. If SMC is requested to perform a protected function then it invokes the security controller to check or authenticate the user which the requesting ACF acts on behalf of. Only if the security handler responds that the check or authentication has been successful, the SMC function will be invoked.

- Merging and splitting of sessions

If two different sessions are merged then the lists of participants are merged and all previous session masters share the session mastership of the new session.

If an existing session shall be split into two new sessions the session master has to provide the two new lists of session participants and the new session masters, and both processing topologies of the new sessions (see chapter 4.2).

- Releasing a session

A session is pulled down by SMC if

- a session master sends a corresponding request,

- timer conditions are met (e.g. concerning the last $\mathrm{ACF}$ request), or

- the only session master leaves the session (e.g. by a system fault).

SMC then releases all existing processing topologies in the session (see chapter 4.2), broadcasts a shut-down notification to all session participants, and deletes the session profile.

\subsection{Processing Topology Handling Functions}

The basic target of SMC is to unify and simplify the usage of multimedia data processing and data transfer capabilities. For this, SMC-API functions are provided to define and manage processing topologies for multimedia data in terms of creating, changing or removing processing entities and their interconnecting communication paths in the context of sessions. The definition of these functions is based on abstractions of the physical layer given in chapter 4.2.1 extending the work introduced in [3]. 
An ACF defining a processing topology and one or more data processing entities of this topology may run on the same end-system (PC or workstation). The difference between them is that the ACF only controls the data processing (via SMC) while at the data processing entity the processing of the multimedia data is performed. So for example, the ACF could look like a console/icon-bar for originating (SMCAPI) commands (e.g. by pressing buttons), while the processing entity (functional node) is the software receiving video data and presenting them in a window.

\subsubsection{Network Abstractions for Session Management and Control}

A data processing entity (DPE) is a piece of hard- and/or software that generates, transforms or consumes multimedia data and is involved in multimedia communication (data transfer) with other DPEs. DPEs are located on end-systems. Examples are video server, microphone or telephone. At the SMC-API, a DPE is abstracted by a functional node (FN). An FN is either a source, a sink, or a relator. A source only generates data units. A sink only consumes data units. A relator both consumes and generates data units. Examples are a microphone as source, a loudspeaker as sink of audio data, and an audio mixer as relator.

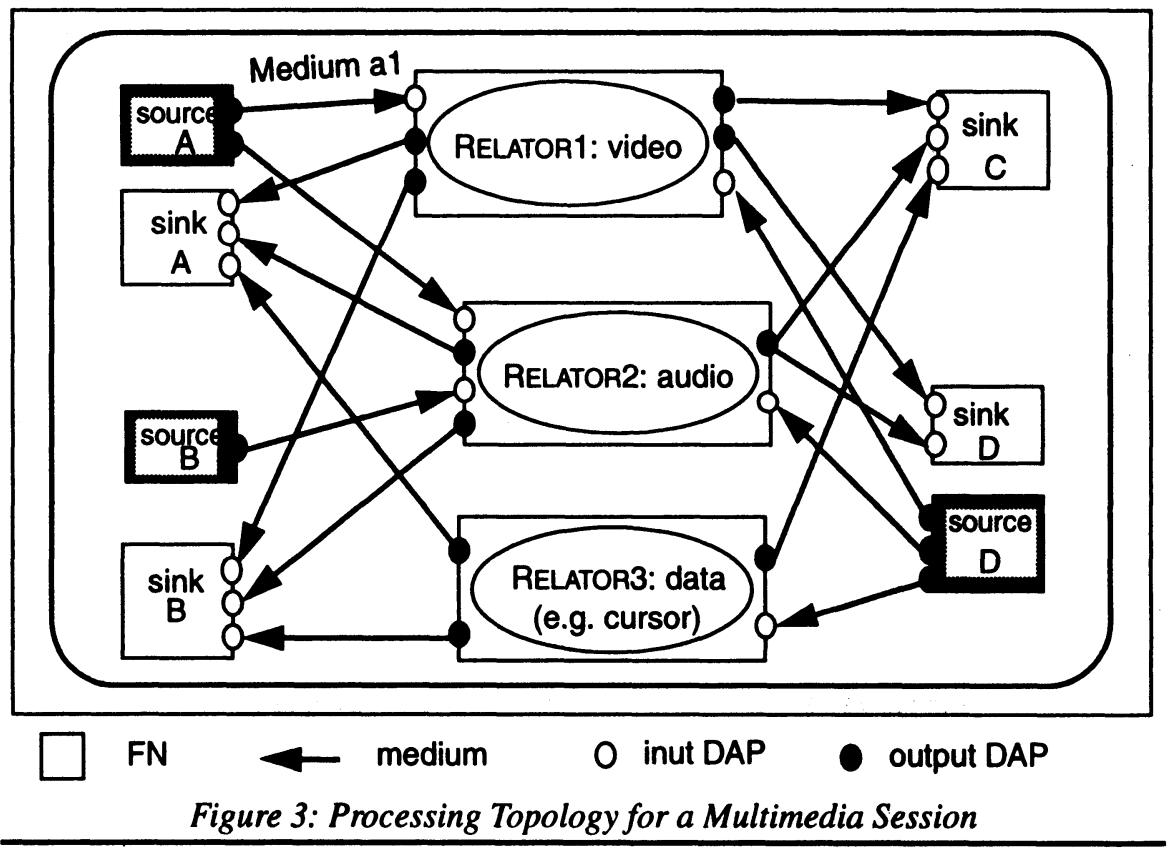

Each FN has a number of data access points (DAP) to access the data. Depending on the direction of data movement, there exist input DAPs and output DAPs. FNs consume data units by reading them from input DAPs and produce data units by writing them to output DAPs. DAPs are typed with the type of data they are able to convey (e.g. audio or video). DAPs may support a single or multiple types. 
A medium is the abstract representation of the data transfer functionality for moving data from one DAP of an FN to another DAP of another FN. The data movement is uni-directional. Multiple media may originate from a single output DAP, while only exactly one medium may end at an input DAP. Multiple media may connect two FNs, e.g. audio and video.

Based on these abstractions, a processing topology is a directed graph given by the directed interconnection of DAPs of functional nodes with media. An example is shown in figure 3. A data path is a path in a processing topology. Data paths always start at output DAPs, may go through relator nodes, and do always ends at an input DAP. A data flow is then the sequential chain of data units that is processed and transferred on data paths.

\subsubsection{Defining and Realizing a Processing Topology}

Each ACF participating in a session can define any number of processing topologies for the session by executing the following steps:

1. create or join a session (see chapter 4.1).

2. announce the new topology to SMC (that returns a unique processing topology identifier to be used as common identification means for all subsequent interactions concerning this topology).

3. define the desired structure of the processing topology, i.e. the nodes and media of the topology, as well as stream control and synchronization relationships and QoS requirements.

4. build up the topology in the actual session.

5. activate and manage controlled streams in the processing topology.

During life-time of a session an ACF can inquire the status of a processing topology (see chapter 4.1), change a topology by adding or removing FNs, re-negotiate QoS parameters (see chapter 4.3), or release a topology.

All FNs and media being available for instantiation are stored in a SMC data base. The maintenance of this data base, e.g. if new data processing and transfer functions are introduced in the overall system (i.e. in the physical layer) is done by corresponding management actions (see chapter 4.5.5).

- Defining functional nodes for the topology graph

Defining a node of a processing topology means to identify

- the type of desired (data) processing function to be realized by the FN To get the unique type name of a processing function, an ACF may interact with a trader function. Trading is a typical middleware functionality outside SMC.

- optionally, a name of the functional node

A functional node name (unique within the session) can be used by (other) ACFs to get the unique identifier of this node from SMC and to avoid duplicate creation of the same node instance. 
- optionally, the location (end-system) of the processing function

The ACF can optionally define the end-system on which the processing function (represented by the FN) shall be executed. If the ACF does not define a location, then SMC determines an appropriate end-system for the FN.

According to this information, an instance of the processing function will be started up by SMC on the determined end-system when building up the processing topology (see below).

- Defining media for the topology

A medium is defined to get an identifier for a data path between two functional nodes and to provide quality of service (QoS) parameters, if no end-to-end QoS handling is realized for a complete data path (see chapter 4.3).

\section{- Defining the linkage of nodes with media}

In this definition step the nodes are interconnected by media. Data movement is directed, so for each medium the source node and the sink node are defined. Each input or output functionality of a node is addressed by a DAP. The actual instantiation of the communication paths abstracted by the media is performed during the build up of the topology.

\section{- Defining streams}

In the context of an application, the control of data processing and transfer functionality by ACFs may also be required during runtime, e.g. to interactively start/ stop a data flow, to alter the speed of a data flow, to play it backwards, or to realize synchronization relationships between data flows. The SMC-API includes stream handling functions for such purposes. To prepare the usage of stream handling functions an ACF has to define the topology or relevant parts of it as stream. The details of SMC stream handling are described in chapter 4.4.

\section{- Building up processing topologies}

Having defined the structure of the desired processing topology, an ACF can request SMC to build up the topology, i.e. to actually instantiate the logical description in the physical network. In that phase SMC starts up the processing functions represented by FNs, instantiates the DAPs, and establishes a network connection for each medium between FNs (this includes QoS negotiation and resource reservation - see chapter 4.3). If there are no streams defined in the processing topology, SMC automatically lifts the blocking of the data generating or consuming functionality of the nodes, such that data transfer becomes active (it then cannot be further influenced via SMC for the complete life-time of the topology, typical application examples are telephone or videophone connections). If there are streams defined in the processing topology, then the stream control code at the FNs (for the desired stream control protocol used by SMC) is instantiated as needed (see chapter 4.4). 


\section{- Changing processing topologies}

To react on dynamic changes in the communication and processing settings of the related applications an ACF can add/remove nodes and/or media to/from a topology, or may re-negotiate QoS parameters. This is done in the same order as for the initial build up of the topology, i.e. the ACF first defines the changes (resulting in SMC database entries) and can then request to actually change the topology.

There can also be automatic topology changes if an ACF leaves a session (by an explicit deregistration or by an erroneous crash). Then SMC automatically shuts down all topology parts belonging to this ACF.

- Releasing processing topologies

A processing topology is pulled down by SMC if an ACF participating in the session sends a corresponding request, or the session the processing topology belongs to is released. To release a processing topology, SMC first blocks the data generating or consuming functionality of the functional nodes, pulls down the network connections and releases the corresponding network resources. Then the functional nodes are shut down and, finally, the processing topology profile is deleted. SMC notifies the topology shut down to all session participants.

\subsection{QoS Negotiation and Resource Reservation Functions}

Quality of Service $(\mathbf{Q o S})$ determines the degree of satisfaction experienced by users of a (multimedia communication) service ([5]). On the highest level of abstraction, QoS may be described in terms of "audio in CD quality" or "video in VHS quality". At the application-middleware interface (i.e. at the SMC-API), generic QoS parameters will be negotiated like "sample rate" for audio or "colour resolution" for video. These parameters then have to be mapped in SMC onto requirements to the physical components like "bandwidth", "processing time", or "buffer size".

In the definition phase for a processing topology (see chapter 4.2.2) the requesting ACF provides end-to-end $\mathrm{QOS}$ requirements referring to data paths or streams of the topology. Examples for possible QoS parameters that can be sent by an ACF are given below. In the build up phase of the topology SMC maps the QoS parameters received from the ACF to corresponding resource parameter and tries to reserve the required resources. End-to-end requirements influence both the data transfer on the related data paths and the data processing at the FNs. If the initial reservation fails, or in case of system performance degradation, or by an explicit $\mathrm{ACF}$ request, QoS (re-)negotiation between $\mathrm{SMC}$ and the $\mathrm{ACF}$ may be required.

Note, QoS handling in general (including QoS specification, resource reservation, and QoS negotiation) is a field of great importance and effort both in the academical and the technical world. However, comprehensive concepts and solutions are not yet available. So some parts of this area still remain for further study. 


\subsubsection{Media Types and QoS Parameters}

Figure 4 shows media types ${ }^{1}$ with QoS parameters to be negotiated between ACF and SMC. The table is mainly taken from [6]. The ACF provides the required QoS parameters to SMC. There are three cases possible:

- QoS parameters may be defined by range. SMC tries to reserve the resources necessary to guarantee the maximum value. Re-negotiation with the ACF is initiated (on runtime) if the minimum value can be no longer guaranteed.

- QoS parameters may also be defined minimum value. SMC tries to reserve the resources necessary to guarantee this minimum. Re-negotiation with the ACF is initiated by SMC (on runtime) if the minimum can be no longer guaranteed.

- If an ACF does not define any value for a QoS parameter then a default value is used that is defined for each QoS parameter. SMC applies the default value as if the ACF had defined the QoS parameter only by a minimum value (see above).

\begin{tabular}{|c|c|c|c|}
\hline Media type & QoS parameter & Example values & Quality characterization \\
\hline \multirow[t]{6}{*}{ video } & \multirow[t]{2}{*}{ frame rate } & $25 \mathrm{fps}$ & PAL \\
\hline & & $60 \mathrm{fps}$ & HDTV \\
\hline & frame size & $\begin{array}{l}>176 * 144 \\
\text { pixel }\end{array}$ & $\begin{array}{l}\text { MMCF QoS class } 1 \text { ("basic } \\
\text { multimedia applications"- [5]) }\end{array}$ \\
\hline & \multirow[t]{2}{*}{ colour resolution } & $1 \mathrm{bit} / \mathrm{pixel}$ & black/white \\
\hline & & 24 bits/pixel & 16 million possible colours \\
\hline & end-to-end delay & $<250 \mathrm{~ms}$ & MMCF QoS class 2 ([5]) \\
\hline \multirow[t]{4}{*}{ audio } & \multirow[t]{2}{*}{ sample size } & 8 bit & telephone voice quality \\
\hline & & 16 bit & CD quality \\
\hline & sample rate & $44.1 \mathrm{kHz}$ & (for 16 bit sample size) \\
\hline & end-to-end delay & $<150 \mathrm{~ms}$ & MMCF QoS class 3 ([5]) \\
\hline \multirow[t]{2}{*}{ data } & bandwidth & $2 \mathrm{Mbps}$ & e.g. for file transfer \\
\hline & end-to-end delay & $<100 \mathrm{~ms}$ & interactive data applications \\
\hline
\end{tabular}

Figure 4: Medium types and QoS parameters

Note, at a higher level of abstraction QoS could be defined by more intuitive terms like "low quality black/white video for browsing". Such a requirement could then be translated into the medium type "video" with e.g. "352*288 pixel, 1 bit/pixel colour, <1s delay, MPEG-1 compression". However, we see the definition of such higher level (user) QoS types and the translation of them in the application layer, i.e. outside SMC.

1. Note, we use the term "medium type" although the end-to-end QoS parameters in general refer to a data flow on a data path possibly via several media (with the same type). 


\subsubsection{Resource Reservation in the Physical Components}

In the build up phase of a processing topology SMC maps the QoS parameters received from the ACF to the corresponding resource parameter in the physical network and tries to reserve the required resources. Such network resource parameters are according to [6] bandwidth of the communication channels, buffer spaces, and CPU processing power. Before QoS parameters can be translated in such resource parameters there may be an intermediate translation within SMC into something like network QoS parameter and device QoS parameter, e.g. latency, bandwidth, delay, and jitter for any relevant data path as well as timing and throughput demands for the FNs.

As a result of the negotiation the elements in the physical layer concerned with processing and communicating real-time critical data have reserved the required resources. This is notified to the ACF. If the reservation fails, then this is notified to the ACF in order to start a re-negotiation.

Resource reservation in the physical components layer is for further study.

\subsubsection{QoS (Re-)Negotiation}

A (re-)negotiation of the QoS arrangements for a topology can be performed between SMC and ACF if the initial reservation fails, if the degradation of system performance makes it impossible to keep the guaranteed QoS, or after an explicit ACF request, if the $\mathrm{Q} O S$ requirements on the application side have changed. In the former two cases SMC informs the ACF that re-negotiation is necessary by a session event notification (see chapter 4.5.4).

The field of QoS re-negotiation is for further study.

\subsection{Stream Control and Synchronization Functions}

The SMC-API stream handling functions enable an ACF to control the data processing and transfer functionality of (parts of) a processing topology during runtime, e.g. to interactively start/stop a data flow, to alter speed/direction of a data flow, or to realize synchronization relationships between data flows and processing entities. Examples are the presentation/processing of data originating from any (digital) storage (e.g. to realize a video playback) or a synchronized video presentation on several end-systems.

Stream control functions concern parts (i.e. subgraphs) of topologies. We call such a subgraph stream. To realize stream handling functions, SMC orchestrates and monitors the processing and transfer of data at all relevant FNs (e.g. by starting the output of a video server and the input of a video window). This requires SMC to control the timing of FN activations by using a stream control protocols.

Stream handling requires advanced SMC-API concepts which we explain in the following. The concepts are partly taken from [7]. 


\subsubsection{Stream Control Concepts}

- Stream Control Identifier (SCI)

When invoking an SMC-API stream handling function the requesting ACF has to identify the stream concerned by the function. For this, we introduce the stream control identifier (SCI) as generic identification means for stream handling functions. An SCI can have two semantics:

- A sink SCI is defined by an input DAP identifier and refers to the topology part covering all data paths between output DAPs of source nodes and the input DAP used as SCI, i.e. a sink SCI concerns all FNs reached in upstream direction from the input DAP used as SCI.

- A group SCI groups other (sink or group) SCIs in an SCI hierarchy. Such an SCI is used to control all related data paths (determined by the sink SCIs on the lowest hierarchy level) by single function calls (see chapter 4.4.2) and provides the means to define synchronization relationships among data flows on the related data paths (see below).

SCIs are defined during the topology definition phase. Note, some stream handling functions require further rules to complete their invocation semantics. For example, to stop a stream identified by a sink SCI requires to stop just those of the relevant active output DAPs that are not active as source for other streams, such that a multicast stream (from one source, e.g. a video server, to several sink nodes) can be stopped at one specific sink node, but kept at all other sink nodes.

\section{- Dimension Attributes of a Stream Content}

Basically, SMC controls the timing of functional nodes in streams. For this, an ACF has to define the temporal dimension of a controlled stream content (e.g. a file or a data flow captured from a camera). The dimension attributes of a controlled stream are described in the following.

A stream content consists of a sequence of data units. Each data unit is associated with a time-stamp defining its positioning at a time axis. The succession of timestamps describes the stream time (given as sequence numbers, e.g. video frame number, or time units relative to real-time, e.g. SMPTE time-stamps). For periodic streams the difference between succeeding time-stamps $(\Delta \mathrm{ST})$ is fixed. For each stream a "normalized" presentation rate is defined that corresponds to the presentation of the data flow with a speed of 1 . The specification is given by the real-time duration $\Delta \mathbf{R T}$ of the data units. This duration corresponds to the stream time difference $\Delta S T$ (see figure 5). The quotient between stream time and real time, i.e. $\Delta \mathrm{ST} / \Delta \mathrm{RT}$ is called ratio. If the stream content is a stored file then it is bounded, i.e. it has a first and a last data unit, called LB (lower bound) and UB (upper bound).

Note, the dimension parameters refer to one stream content (e.g. a stored file) presented at a sink FN. If the content changes (e.g. to another file), then it can be necessary to re-define the dimension parameters. 


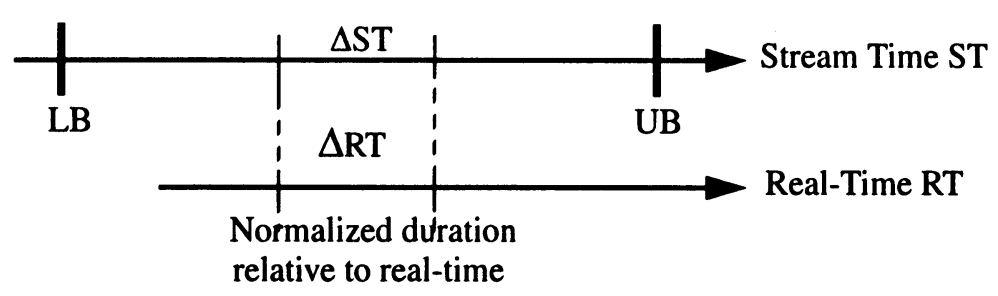

Figure 5: Attributes of a Stored Data Flow

- The Normalized Real-Time-Line (nRT)

The basic means to enable synchronization of streams (e.g. audio/video synchronization or synchronized play-out of the same content at different sinks) is the normalized real-time-line (nRT). This is a real-time-line with the default start point ' 0 '. Each $\mathrm{SCI}$ has a normalized time-line.

In the first step, the contents of a stream are aligned to the nRT of the sink SCI where they shall be presented (aligning a sequence of contents is the basics for play-lists). The alignment of contents is done by reference points, i.e. a point in the content's stream time referring to a point on the nRT. With the content's ratio, for each point in stream time the belonging point on the nRT can be calculated by

$$
m_{n R T}=\left(m_{S T}-P_{S T}\right) \cdot \frac{\Delta R T}{\Delta S T}+P_{n R T}
$$

where $P_{S T}$ and $P_{n R T}$ define reference points and $m_{S T}$ is a point in stream time for which the point $m_{n R T}$ on the $n R T$ shall be calculated.

The play-out at different sinks can be synchronized (e.g. audio and video, or video for cooperative viewing). For this, the nRTs of the sink SCIs are synchronized via $\mathrm{nRT}$ of a group SCI grouping the sink SCIs, i.e. sync relationships are implicitly defined. If e.g. audio and video contents are synchronized, both nRTs of the two sink SCIs are synchronized to nRT of the SCI grouping these sink SCIs by reference points.

\section{- Presentation Attributes of a Stream}

When presenting a stream, the content's stream-time-line or the nRT to which the content is aligned and the time axis of global real-time are fixed with each other ("start on $12 \mathrm{~h} 00$ with frame 1"). This is defined by the reference points between stream-time/nRT and global real-time, describing the presentation start point.

To describe the temporal dimension of the stream presentation moreover the speed factor $\mathbf{S}$ of the presentation is needed. It describes the mapping between $\mathrm{nRT}$ and global real-time. A negative value defines a presentation in reverse direction. How speed values other than " 1 " are either achieved (e.g. by changing the data rate or by dropping/duplicating of data units) depends on the stream control protocol. 


\subsubsection{Stream Handling Functions}

\section{- Controlling Stream Presentation}

SMC-API stream handling functions enable to control data flows during runtime by providing VCR like operations, e.g. to interactively start/stop a data flow, to seek in a stream content, or to change the speed of a data flow.

If one of the corresponding stream handling function is invoked with an sink SCI as stream identifier then the related data path is determined as described above. If an SCI grouping other SCIs is used then the function is propagated through the complete (sub)hierarchy of this SCI and, therefore, executed for every data flow on a data path related to an sink SCI being a leaf of this hierarchy. In the simplest case (without a synchronization relationship) the propagation of such an invocation means to execute this function in parallel for all these data flows without any synchronization between the executions.

\section{- Synchronization of Data Flows}

To control different data paths in a synchronized manner, e.g. a video and an audio data flow basically means that at more than one input DAP data, transferred on the data paths, shall arrive synchronized to each other. For this, SMC supports the definition of so called 'sync relationships' for sink SCIs. The definition of such relationships is done as follows:

- First, all related contents have to be aligned to their sink SCI's nRTs.

- A group SCI for the related group of sink SCIs has to be introduced.

- Then each of the sink SCI nRTs must be synchronized to the group SCI's $\mathrm{nRT}$, i.e. one sync definition per sink SCI (for the related streaming concepts see chapter 4.4.1).

SMC uses these attributes on runtime to propagate the related function invocations in an appropriate manner to guarantee the required synchronization parameters.

\section{- Handling of Composed Streams}

Processing continuous data, such as in news studio environments, require runtime capabilities to concatenate and combine stream contents from different sources (files) to form a resulting data flow. We call the content parts clips and the resulting data flow composed stream. The latter is represented by a meta definition (also called 'Edit Decision List') which defines the temporal relationships between the clips, i.e. the order in which the clips are concatenated (and possibly special transition effects between clips like overlaying). Furthermore, the meta definition contains the locations (servers) where the source files of the clips are stored. Note, in many cases, the meta definition is the only representation of a composed stream, i.e. this data flow is not actually stored as (new) data material, but has to be established on runtime. SMC provides the means to handle such meta definitions on runtime in a way that an ACF can control a composed stream via the SMC-API as if it were a single physical data flow. 


\subsection{Further SMC Function Groups}

\subsubsection{ACF Concurrency and Coordination Support Functions}

In distributed applications, ACFs need means to coordinate and synchronize their concurrent actions. Examples are the synchronization of ACF states and the coordination of access to resources. SMC offers concurrency and coordination support functions to provide powerful means for these purposes. The underlying concepts are mainly taken from the multipoint communication service specification in the T.120 standard series ([4]). The basic one is the concept of tokens. A token represents an information item (e.g. an access right) in the context of an application. Tokens are created and specified by ACFs during runtime and can be grabbed, given back, or checked by ACFs. The possession of a token can be exclusive or non-exclusive. The semantics of a token is not visible to SMC. SMC only maintains the existing tokens according to their specification and provides the functions enabling ACFs to create, request, take, pass, delete, ... tokens.

\subsubsection{ACF-ACF Communication Support Functions}

Application control functions are located on different end-systems. In the context of distributed applications, the ACFs need to be able to exchange information. This ACF-ACF communication is facilitated by the communication support functions of SMC enabling ACFs to send information items (e.g. a message string) via $S M C$ to other ACFs.

Before such a request both ACFs must be participants of the same session. The exception is sending information to a permanent application control function (PACF). A PACF is a specific ACF permanently waiting for SMC notifications. PACFs are necessary since, in general, ACFs are not permanently running on their end-systems. In the course of an application, it may be required for ACFs to invoke or inform other ACFs that are not yet running. At most one PACF exists on an endsystem. A PACF is uniquely identified by its globally unique end-system address. Depending on the specific end-system a PACF belongs to, it has the capability to appropriately react on requests, e.g. to cause an $\mathrm{ACF}$ to join a session.

SMC enables to address individual ACFs, the PACF of an end-system, all ACFs participating in a session, as well as predefined groups of ACFs. Speedy and ordered information delivery is supported by various transfer semantics, such as 'unreliable delivery' and 'reliable FIFO delivery'.

Note, the communication support functions are provided in addition to the processing topology handling functions as described in chapter 4.2. A separation of the ACF-ACF communication from the concepts for processing and transfer of multimedia data mainly results from the different layers of abstractions. ACF-ACF communication is among entities layered on top of SMC. In contrast, the multimedia data are transferred between the data processing entities (see figure 2). 


\subsubsection{ACF-FN Communication Support Functions}

Communication among ACFs and functional nodes may be required, e.g. for directly controlling FN settings (e.g. to change window settings of a video sink, or to define parameters for a relator node performing overlaying effects). To enable ACF-FN communication, SMC provides the necessary address information to ACFs. The exchange of information between ACFs and FNs is outside SMC. It can be realized e.g. via CORBA mechanisms ([8]).

\subsubsection{Session Event Handling Functions}

In the context of an application, it might be important for an ACF to get informed about the occurrence of events concerning the status of a session. The SMC-API contains functions that enable an ACF to define events it wants to be informed about and corresponding notifications sent by SMC when such an event occurs. Session event handling can be based on an abstract model of state transitions within a session (by a finite state machine like the IN-Basic Call State Model) including a definition of all events that trigger a runtime notification by SMC. Examples are:

- QoS degradation, i.e. if QoS descends below the guaranteed limiting value (e.g. the lower margin of the guaranteed QoS range).

- Session administration notifications, like changing the session master.

- Stream events, like reaching the end of a file.

- Error events, like the crash of a processing entity. Error events may be automatically notified to the session master ACF.

\subsubsection{Functions for SMC Management}

In an overall multimedia system, configuration management functionality is required to setup, configure, or delete entities both in the application and in the physical layer. This basically means to maintain the corresponding data bases, used e.g. by SMC, when new processing entities are installed or users are subscribed. The SMC management should include functions to define or remove data processing entities in the SMC environment, i.e. store/delete the corresponding data (like address information) in/from the relevant SMC data bases. Furthermore, it should enable introducing or removing $A C F s$ in the SMC environment.

\section{EXAMPLE SCENARIO}

The example describes cooperative and synchronous viewing of a video. The same video is played out to two users at two different end-systems. Figure 6 shows the processing topology. The video is played out by a video server and transferred to two video sinks that are located on the end-systems of the users. The stream has an $1: n$-multicast structure. Both users are enabled to manipulate the presentation of the video. Between the two data flows a sync relationship is defined to make sure both users view the same data at the same time. Figure 7 reflects the basic information flows. Of course, not all details of the example scenario can be shown. 


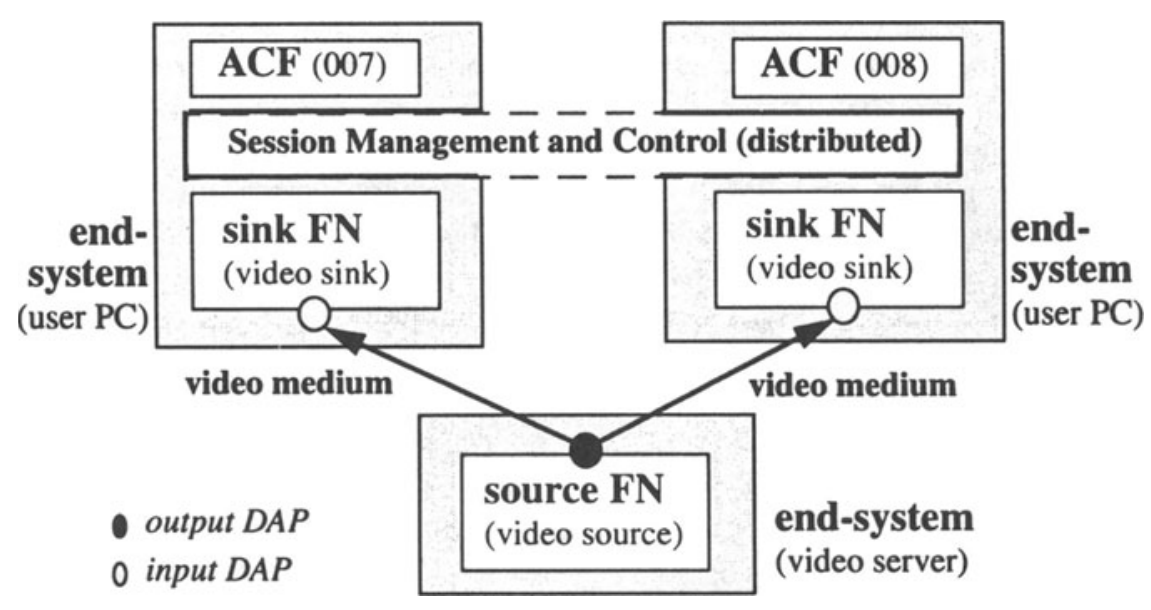

Figure 6: Example Scenario - Cooperative Viewing of a Video

The actions listed below refer to the boxes in figure 7 .

1. A user starts an application (represented by ACF '007') to view video files from a server. In the initial step a session is created. The only user interaction may happen to identify the desired video server. All the following steps (till action 8) are then done automatically by the ACF.

2. SMC sets up the session, i.e. a session profile is created, the requesting ACF is stored as session master and first participant, and the initial attributes (e.g. 'masterShare' is "true" to allow later cooperation) are stored. SMC generates a new session identifier and returns it to the ACF.

3. The processing topology definitions for $\mathrm{ACF}$ ' 007 ' are done, i.e. the definition of the source functional node and the sink node on the end-system (PC) of ACF ' 007 ' linked by a medium defined for video. SMC creates a topology profile and a new processing topology identifier. It checks the functional node and medium definitions and stores it in the topology profile.

4. The stream (SCI) definitions for ACF '007' are done. First, the basic sink SCI is defined (i.e. requested by the ACF and generated by SMC). Then, a group SCI is defined in advance to be the common SCI for all ACFs joining the session. An alternative solution would be the second ACF participating in the session defines the group SCI when it joins the session and sends the necessary information to the first ACF via ACF-ACF communication (see action 12).

After the definition phase the ACF requests for the actual instantiation of the processing topology. This may be done automatically by the ACF, i.e. without interaction between user and application.

5. SMC performs the necessary steps to instantiate the processing topology, i.e. it starts up the functional nodes (i.e. the processing functions they represent), instantiates the DAPs, establishes a connection for the medium between the nodes. Since the defined topology represents a "controlled streams", the nodes 


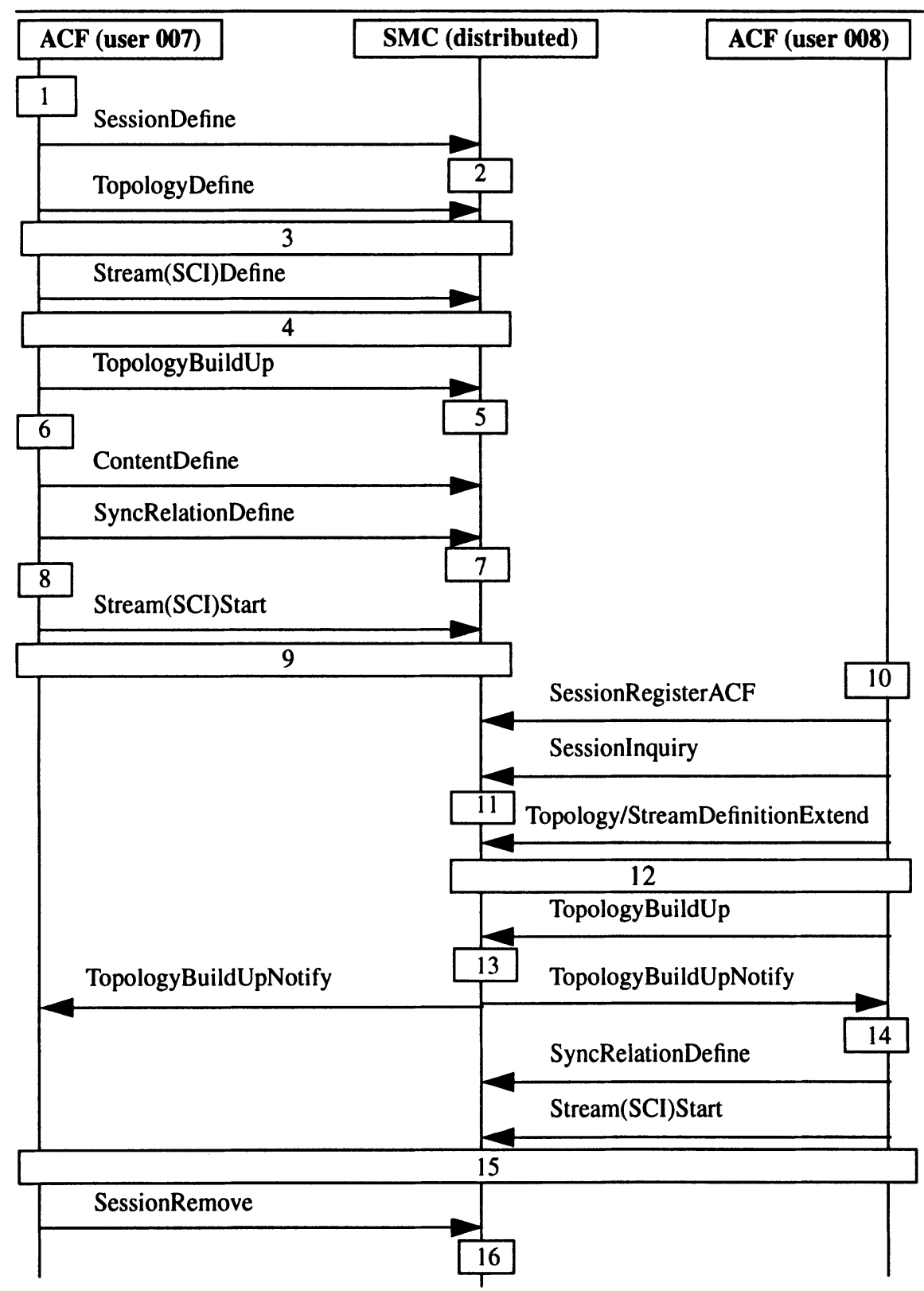

Figure 7: Basic Information Flows for the Example Scenario

keep initially blocked (see chapter 4.4.1). After these steps SMC notifies the success of the topology realization to the ACF.

6. The ACF defines the content to be played out, e.g. after the application logic performed a dialogue with a user to manually select a file. The ACF aligns the 
content to the sink SCI's nRT and defines the sync relation between the nRTs of sink and group SCI (see chapter 4.4.1).

7. SMC stores the dimension parameter (ratio, lower and upper bound) for the (video) content, the alignment to the sink's nRT, and the reference point between the video sink SCI's nRT and the group SCI's nRT (to prepare for later synchronization with other sinks).

8. After having finished the definitions, the ACF can request the activation of the play-out (e.g. after the user pressed the start button). A technical opportunity is to request an explicit content pre-load first.

9. Having received the start request for the stream SMC lifts the blocking of the nodes, such that the data transfer becomes active and the video presentation at the sink node is started. In the following the user (represented by ACF '007') works with the video (start, stop, seek, etc.).

10. Another user starts an application (represented by ACF '008') to cooperatively work with the first user, i.e. to view the video files together. The ACF registers for the session and queries about the existing topology to be able to define the appropriate extensions. Note, SMC is a distributed function, such that the new ACF does not communicate with the same SMC entity as the first ACF. However, the SMC distribution is not visible to the ACFs.

11. SMC stores ACF '008' as new participant and additional master for the session and returns the complete topology profile as response to the query.

12. The topology extensions for ACF '008' are defined. This includes the definition of the sink node on the end-system (PC) of ACF '008' and the linkage to the source node by a medium defined for video. Furthermore, the second sink SCI is defined. Then, the instantiation of the extended topology is requested.

13. SMC starts up the new sink functional node, instantiate its input DAP, and establishes a connection for the medium between the source and the new sink node. The multicast from the source node to the new sink keeps initially blocked. After these steps SMC confirms the actions to both ACFs.

14. To enable synchronized played-out between the sinks, the new sink SCI's nRT must be synchronized to the group SCI's nRT in exactly the same way as it was done for the other sink (see action 7). After all definitions are successfully completed the ACF requests for the activation of the stream. For the start command the group SCI is used with a special start time value ("current") such that the data actually played out at the source are multicasted also to the new sink node. Since the first sink node is already active nothing happens for it.

15. SMC now lifts the blocking of the multicast from the source node to the new sink and the blocking of this sink, such that the data transfer becomes active and the video presentation at the new sink node is started.

In the following, the cooperative work of the users happens. They may select further videos, start/stop the presentation, seek in a file, etc. At the end, the 
users decide to close the session. In this scenario, ACF '007' sends the release request. ACF ' 008 ' does not need to explicitly deregister from the session.

16. SMC releases the session and deletes the session profile. This includes an automatic shut-down and remove of the complete topology.

\section{CONCLUSIONS}

To close the gap between the functionality offered by basic communication and operating systems and the requirements of interactive, distributed multi-user multimedia applications, generic middleware functions should be provided via application-oriented programming abstractions. Based on our Multimedia Reference Model as an overall conceptual framework we introduced session management and control (SMC) as a special middleware part to unify and simplify the usage of data processing and data transfer capabilities in the physical layer. We explained motivation and concepts of the basic groups of SMC functionality reflecting many areas currently under consideration in the academical and the technical world, like quality of service and stream handling. For illustration, we gave an example scenario. On the basis of our concepts a detailed SMC protocol specification is possible.

\section{REFERENCES}

[1] Abramowski, St., Elixmann, M., Gappisch, H., Heister, U., Heuter, U., Klabunde, K., "A Service Creation Environment for Intelligent Networks", Proceedings of the Int. Zurich Seminar on Digital Communication, March 16 - 19, 1992, Zurich, Switzerland

[2] Elixmann, M., de Greef, B.L., Lelkens, A.M.M., Neunast, K.W., Tjabben, H., "Open Switching - Extending Control Architectures to Facilitate Applications", Proceedings of the ISS '95, Berlin, Germany, April 23 - 28, 1995

[3] Abramowski, St., Klabunde, K., Konrads, U., Neunast, K.W., Tjabben, H., "The Multimedia Reference Model: A Framework Facilitating the Creation of Multi-User, Multimedia Applications", IFIP-TC6 Working Conf. on Intelligent Networks, Copenhagen, Aug. 30-31, 1995

[4] ITU-T Study Group 8, "Multipoint Communication Service for Audiographics and Audiovisual Conferencing - Service Definition, Recommendation T.122"

[5] Multimedia Communications Forum "Multimedia Communications Quality of Service - Framework - Multimedia Desktop Collaboration Requirements", MMCF/95-010

[6] Nahrstadt, K., "An Architecture for End-To-End Quality of Service Provision and its Experimental Validation", PhD thesis, Univ. of Pennsylvania, 1995

[7] Rothermel, K., Helbig, T., "Clock Hierarchies: An Abstraction for Grouping and Controlling Media Streams", IEEE Journal on Selected Areas in Communications, Special Issue on Synchronization Issues in Multimedia Communications Vol. 14, No. 1, January 1996

[8] Object Management Group, "CORBA services: Common Object Services Specification”, OMG Document No 95-3-31, March 1995 